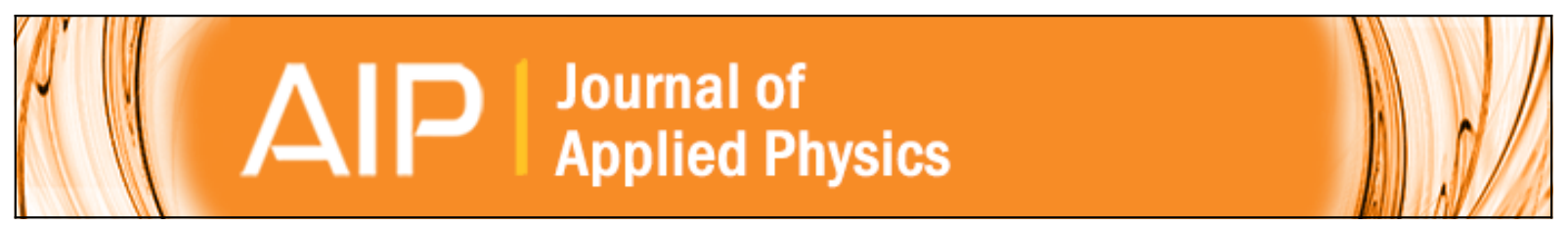

\title{
Disorder driven structural and dielectric properties of silicon substituted strontium titanate
}

Sita Dugu, Shojan P. Pavunny, Yogesh Sharma, James F. Scott, and Ram S. Katiyar

Citation: Journal of Applied Physics 118, 034105 (2015); doi: 10.1063/1.4927042

View online: http://dx.doi.org/10.1063/1.4927042

View Table of Contents: http://scitation.aip.org/content/aip/journal/jap/118/3?ver=pdfcov

Published by the AIP Publishing

\section{Articles you may be interested in}

Structure, dielectric, and magnetic properties of $\mathrm{Sr} 2 \mathrm{TiMnO} 6$ ceramics

J. Appl. Phys. 108, 094108 (2010); 10.1063/1.3500369

Structural, electrical, and magnetic properties of multiferroic Bi $1-x$ La x Fe 1 - y Co y O 3 thin films J. Appl. Phys. 107, 124109 (2010); 10.1063/1.3437232

Effect of $\mathrm{Nd}$ modification on electrical properties of mixed-layer Aurivillius phase Bi 4 Ti 3012 -SrBi 4 Ti 4 O 15 J. Appl. Phys. 102, 024102 (2007); 10.1063/1.2753582

Tunability and ferroelectric relaxor properties of bismuth strontium titanate ceramics

Appl. Phys. Lett. 90, 182902 (2007); 10.1063/1.2734958

Dielectric properties of layered perovskite $\mathrm{Sr} 1-\mathrm{x} \mathrm{A}$ x Bi $2 \mathrm{Nb} 2 \mathrm{O} 9$ ferroelectrics ( $\mathrm{A}=\mathrm{La}, \mathrm{Ca}$ and $\mathrm{x}=0,0.1$ ) Appl. Phys. Lett. 76, 2934 (2000); 10.1063/1.126521

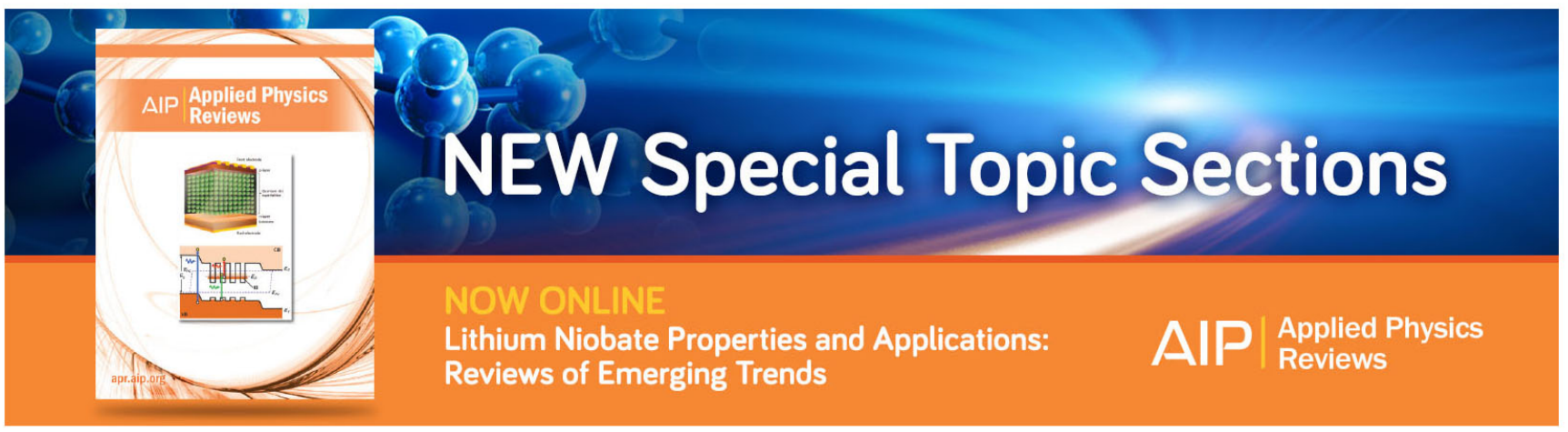




\title{
Disorder driven structural and dielectric properties of silicon substituted strontium titanate
}

\author{
Sita Dugu, ${ }^{1}$ Shojan P. Pavunny, ${ }^{1, a)}$ Yogesh Sharma, ${ }^{1}$ James F. Scott,,${ }^{1,2}$ \\ and Ram S. Katiyar ${ }^{1, a)}$ \\ ${ }^{1}$ Department of Physics and Institute for Functional Nanomaterials, University of Puerto Rico, \\ P.O. Box 70377, San Juan, Puerto Rico 00936-8377, USA \\ ${ }^{2}$ Department of Physics, Cavendish Laboratory, University of Cambridge, Cambridge CB3 OHE, \\ United Kingdom
}

(Received 11 May 2015; accepted 7 July 2015; published online 17 July 2015)

\begin{abstract}
A systematic study on structural, microstructural, optical, dielectric, and electrical properties of phase-pure silicon-modified $\mathrm{SrTiO}_{3}$ polycrystalline electroceramics synthesized using high energy solid state reaction techniques is presented. The asymmetry and splitting in the X-ray diffractometer spectra and the observation of first order transverse optical $\mathrm{TO}_{2}$ and longitudinal optical $\mathrm{LO}_{4}$ modes in Raman spectra (nominally forbidden) revealed the distortion in the cubic lattice as a result of breaking of inversion symmetry due to doping. A bandgap $\mathrm{E}_{\mathrm{g}}$ of $3.27 \mathrm{eV}$ was determined for the sample by diffuse reflectance spectroscopy. A high dielectric constant of $\sim 400$ and very low dielectric loss of $\sim 0.03$ were obtained at $100 \mathrm{kHz}$ near ambient conditions. The temperature dependence of the dielectric data displayed features of high temperature relaxor ferroelectric behavior as evidence of existence of polar nano-regions. The ac conductivity as a function of frequency showed features typical of universal dynamic response and obeyed a power law $\sigma_{a c}=\sigma_{d c}+A \omega^{n}$. The temperature dependent dc conductivity followed an Arrhenius relation with activation energy of $123 \mathrm{meV}$ in the $200-500 \mathrm{~K}$ temperature range. The linear dielectric response of $\mathrm{Pt} / \mathrm{SrSi}_{0.03} \mathrm{Ti}_{0.97} \mathrm{O}_{3} / \mathrm{Pt}$ dielectric capacitors was well characterized. The measured leakage current was exceptionally low, $13 \mathrm{nA} / \mathrm{cm}^{2}$ at $8.7 \mathrm{kV} / \mathrm{cm}$, revealing an interface blocked bulk conduction mechanism. (C) 2015 AIP Publishing LLC. [http://dx.doi.org/10.1063/1.4927042]
\end{abstract}

\section{INTRODUCTION}

Silicon technology, driven by Moore's law, demands new material systems to realize fast switching and low power consumption for higher density logic and memory devices. In this regard, strontium titanate (STO) is one of the most investigated perovskite $\left(\mathrm{ABO}_{3}\right)$ oxides, due to its superior characteristics $^{1-3}$ such as high dielectric constant $\varepsilon^{\prime}$ and quality factor $Q$, incipient ferroelectricity, good chemical stability, high breakdown strength and large optical bandgap, which permit technological applications ${ }^{4,5}$ in microelectronics and photonics such as charge storage layers in dynamic random access memories (DRAM), insulating sheets in resistive random access memories (RRAM), gate oxide films in metal-oxide-semiconductor field-effect transistors (MOSFET), voltage controlled tunable permittivity sheets in microwave devices, and buffer layers in optical waveguides. The growth of epitaxial perovskite oxide thin films on silicon paved the way for an entirely new device physics based on utilization of the anisotropic response of crystalline oxide films grown on lattice-matched semiconductor substrates. ${ }^{6}$ In this context, it is worth noting that STO and its doped variants can grow well on an Si (100) substrate with very little lattice mismatch to provide a clean/ abrupt interface, a higher uniformity, minimal defect density,

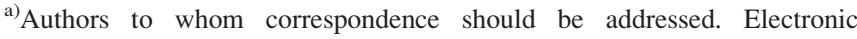
addresses: shojanpp@gmail.com and rkatiyar@hpcf.upr.edu. Tel.: 787751 4210, Fax: 7877642571.
}

and lower leakage currents compared with polycrystalline or amorphous metal oxide films for various potential device applications.

The dielectric and other related physical properties of the quantum paraelectric STO can be tuned by doping as well as by substituting isovalent or heterovalent ions into $\mathrm{Sr}$ and/or Ti sites. ${ }^{7,8}$ The present work focuses on the effect of Si doping in STO and is stimulated by its potential role in oxide electronics. The ability to control structural, dielectric, and transport properties of STO by doping ${ }^{9}$ is an important step towards the design of various microelectronic devices such as capacitors, MOSFETs, thermistors, and varistors. The effect of doping STO with neutral acceptor (isovalent) $\mathrm{Si}$ (of stable valency $4+$ ) at the Ti site and the rich physical properties of the electroceramics obtained are presented in this paper.

\section{EXPERIMENTAL}

$\mathrm{SrSi}_{0.03} \mathrm{Ti}_{0.97} \mathrm{O}_{3}$ (SSTO) powders were synthesized by a high-energy solid state reaction from a stoichiometric mixture of $\mathrm{SrCO}_{3}$ (99.99\%), $\mathrm{TiO}_{2}$ (99.9\%), and $\mathrm{SiO}_{2}(99.995 \%)$. Mechanical ball milling of stoichiometric amounts of precursors was carried out for $10 \mathrm{~h}$ in a methanol medium for fine mixing. After drying the mixture was calcined at $800^{\circ} \mathrm{C}$ for $2 \mathrm{~h}$ and then at $1300^{\circ} \mathrm{C}$ for $6 \mathrm{~h}$ using a Carbolite HTF1700 furnace with a heating and cooling rate of $5^{\circ} \mathrm{C} / \mathrm{min}$. The synthesized powders were pressed in the form of thick pellets (13 mm diameter and $1.03 \mathrm{~mm}$ thickness) with $7 \mathrm{wt} . \%$ 
polyvinyl alcohol at a uniaxial pressure of 4 tons and later sintered at $1350{ }^{\circ} \mathrm{C}$ for $4 \mathrm{~h}$. Phase purity of the sample was checked in a slow scan mode $\left(0.25^{\circ} / \mathrm{min}\right)$ with a Rigaku Ultima III x-ray diffractometer (XRD) equipped with $\mathrm{CuK} \alpha$ radiation $(\lambda=1.5405 \AA$ ) source operating in Bragg-Brentano $(\theta-2 \theta)$ geometry at $40 \mathrm{kV}$ and $40 \mathrm{~mA}$. The sample was then subjected to temperature dependent Raman spectroscopy studies. The laser line at $514.5 \mathrm{~nm}$ from a Coherent Argon ion laser (Innova 70-C) was focused on the sample. A liquidnitrogen-cooled CCD device collected the Raman scattered signal through a $50 \mathrm{X}$ objective. We collected low temperature spectra of the sample in vacuum from $80 \mathrm{~K}$ to $300 \mathrm{~K}$ using a Linkam module. Room temperature surface topography of the sample at $3500 \times$ and $10000 \times$ magnifications was analyzed in vacuum using a scanning electron microscope (SEM) having a resolution better than $1 \mu \mathrm{m}$. Elemental analysis of the pellet was carried out by recording the energydispersive $\mathrm{X}$-ray (EDX) spectra. X-ray fluorescence (XRF) spectra were collected to identify and to determine the concentrations of the elements present in the sample. For dielectric and electrical characterization, the synthesized pellet was DC sputtered at room temperature with $\mathrm{Pt}$ to form the top (5.4 $\mathrm{mm}$ diameter) and bottom (13 $\mathrm{mm}$ diameter) electrodes. The resulting MIM structures were annealed at $400{ }^{\circ} \mathrm{C}$ in oxygen ambient for the proper adhesion of $\mathrm{Pt}$ and for the recovery of any possible sputter damage. The dielectric and DC leakage current measurements were done under vacuum $\left(\sim 10^{-6}\right.$ Torr $)$ using an HP4294A Impedance Analyzer and Keithley electrometer (model \#6517 A). The samples were kept in the dark during dielectric characterization. Temperature control was achieved using a programmable temperature controller [MMR Technologies].

\section{RESULTS AND DISCUSSION}

\section{A. Structural and elemental analysis}

Figure 1 shows selected high resolution Miller indexed Bragg peaks [(100), (110), and (200)] of polycrystalline SSTO powder XRD spectra collected at room temperature, which satisfy a cubic crystal structure ${ }^{10}$ belonging to space group $\operatorname{Pm} \overline{3} m\left(O_{h}^{1}\right)$ no. 221 , where the cations $\mathrm{Ti}^{4+} / \mathrm{Si}^{4+}$ are sixfold-coordinated $(Z=6)$ and $\mathrm{Sr}^{2+}$ are twelvefoldcoordinated $(Z=12)$ by $\mathrm{O}^{2-}$ anions. The observed asymmetry and splitting in these peaks reveal an appreciable distortion in the cubic lattice as a result of silicon doping. The presence of elements in the ceramic was analyzed by XRF and EDX methods. Figure 2 is a representative EDX spectrum of the SSTO pellet excited by an electron beam of energy $20 \mathrm{kV}$, showing the presence of elements $(\mathrm{Sr}, \mathrm{Si}, \mathrm{Ti}$, and $\mathrm{O}$ ) making up the oxide compound along with their respective characteristic $\mathrm{x}$-ray emission lines $(\mathrm{O} \mathrm{K} \alpha$ $0.525 \mathrm{keV}$, Si K $\alpha 1.74 \mathrm{keV}$, Si K $\beta 1.83 \mathrm{keV}$, Sr L $\alpha 1.80 \mathrm{keV}$, Sr L $\beta 1.872$, Ti K $\alpha 4.51 \mathrm{keV}$, and Ti K $\beta 4.93 \mathrm{keV}$ ). On the average, the Sr:Ti:Si atomic ratio, estimated from the intensities of the characteristic lines, was in good agreement with the composition stoichiometry of SSTO precursors prior to annealing. A typical SEM micrograph of the sintered pellet depicted in the inset of Fig. 2 demonstrates well defined granular structure with an average grain size of about

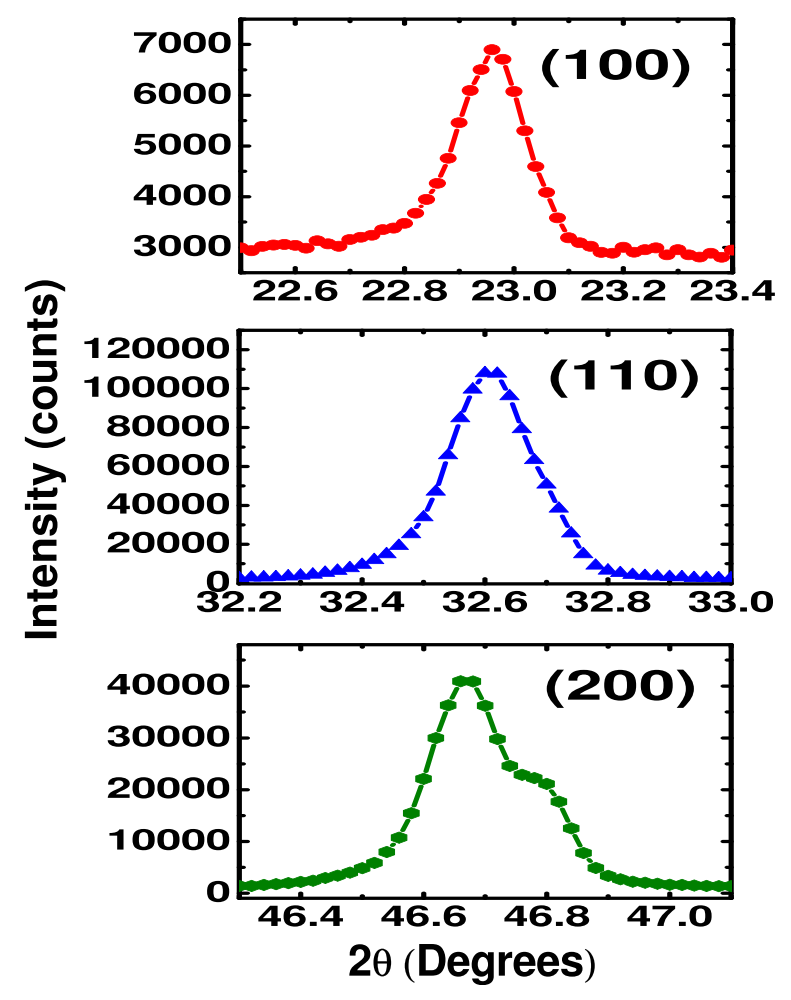

FIG. 1. Selected room temperature powder XRD Bragg peaks of cubic SSTO polycrystalline ceramics.

$\sim 1-2 \mu \mathrm{m}$ and shows the presence of pores. XRF data (not shown) revealed the presence of strontium, titanium, silicon and oxygen with $50.2 \%, 25.7 \%, 0.173 \%$, and $24 \%$, respectively, by mass, which is in fairly good agreement with our theoretical stoichiometry: i.e., $7.1862 \mathrm{~g}, 3.808 \mathrm{~g}, 0.0691 \mathrm{~g}$, and $3.93653 \mathrm{~g}$, respectively.

\section{B. Raman spectroscopy}

Figure 3 shows the unpolarized temperature dependent $(80-300 \mathrm{~K})$ Raman spectra of random textured SSTO ceramics. At room temperature, no first-order Raman modes are expected in SSTO on the basis of the factor group

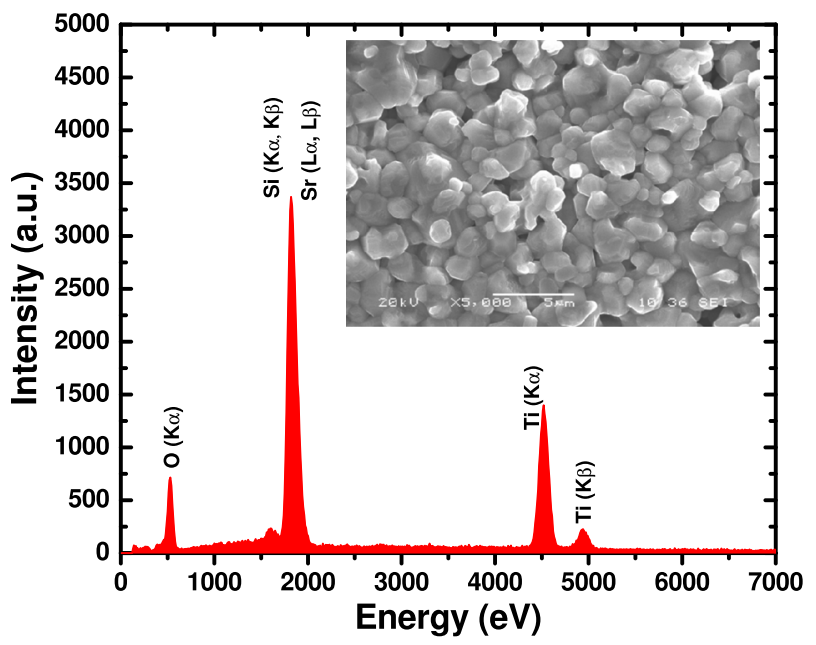

FIG. 2. EDX spectrum of SSTO polycrystalline sample recorded at room temperature. SEM micrograph of the sintered pellet is given in the inset. 


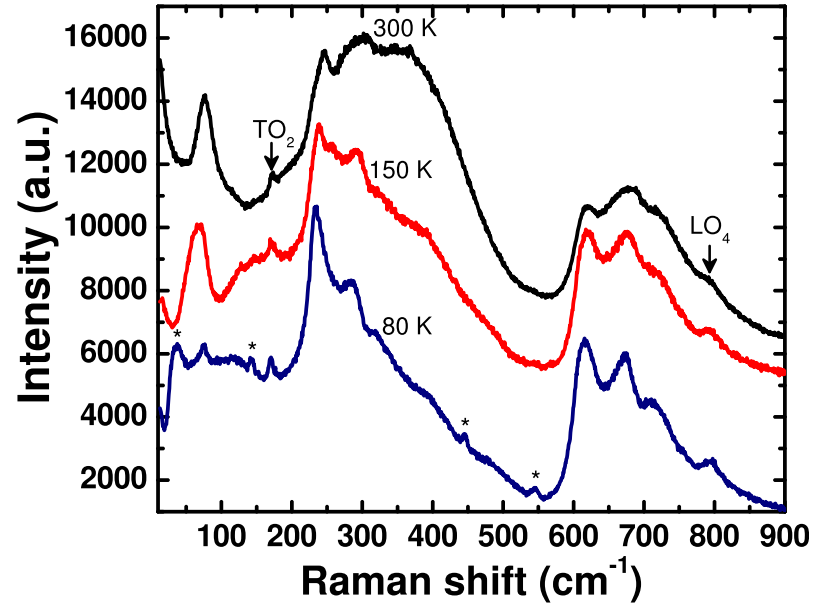

FIG. 3. Temperature dependent Raman spectra of SSTO ceramics.

symmetry analysis since all five atoms in the unit cell of the cubic crystal structure, belonging to space group $\operatorname{Pm} \overline{3} m\left(O_{h}^{1}\right)$, lie on the inversion centers. The normal mode analysis for the cubic symmetry is given by $\Gamma=3 F_{1 u}^{\text {Optic }}+1 F_{2 u}^{\text {Optic }}$ $+1 F_{1 u}^{\text {Acoustic }}$, where one $F_{1 u}$ symmetry mode belongs to acoustical mode and the remaining three $F_{1 u}$ modes and one $F_{2 u}$ mode are optical modes and are Raman inactive (odd parity). Each of the $F_{1 u}$ optical modes further splits into pairs of LO (longitudinal optic) and TO (transverse optic) modes as a result of long range electrostatic fields present in the ionic dielectric ( $F_{2 u}$ is "silent" with no infrared activity or LO/TO splitting). It is generally understood that the observed room temperature continuous spectra (no sharp, discrete lines) (Fig. 3) are second-order Raman scattering that involves the creation or destruction of two phonons in the scattering process. $^{11,12}$ The observed spectra consist of a low frequency band at $79 \mathrm{~cm}^{-1}$ and two second-order broad bands centered in the $200-400$ and $600-800 \mathrm{~cm}^{-1}$ regions. The numerical values of the peak frequencies of each mode determined from experimental spectra are given in Table I along with their possible mode assignments. Although broad features generally arise from large wave-vector modes near the Brillouin zone boundary, where the density of states is large, relatively sharp features can arise from sum and difference combinations of the long wavelength vibrations, typically labeled as $\mathrm{TO}_{1}, \mathrm{TO}_{2}, \mathrm{LO}_{1}$, etc., for transverse and longitudinal optical modes of increasing energy (4 each). TA designates transverse acoustic mode and it has lower energy than the LA (longitudinal acoustic) mode at the same wave vector (or at the zone boundary). Strictly speaking only at small wave vector the acoustic phonons are well characterized as TA and LA. The terminology, however, is also referred to as TA and LA phonon branches at the zone boundary. The band at $79 \mathrm{~cm}^{-1}$ is made up of the difference combinations $\mathrm{TO}_{2}-\mathrm{TA}$ and $\mathrm{TO}_{2}-\mathrm{TO}_{1}$, whereas the one at $250 \mathrm{~cm}^{-1}$ is assigned to be the overtones $2 \mathrm{TA}$ and $2 \mathrm{TO}_{1}$ and the combination $\mathrm{TO}_{1}+\mathrm{TA}$. The single phonon energy of TA (zone boundary) and $\mathrm{TO}_{1}$ (zone center) can each be estimated to be $125 \mathrm{~cm}^{-1}$ from the position of the latter band. The deconvoluted band at $307 \mathrm{~cm}^{-1}$ is composed of $\mathrm{TO}_{2}+\mathrm{TA}, \mathrm{TO}_{2}+\mathrm{TO}_{1}$, and $\mathrm{TO}_{4}-\mathrm{TO}_{2}$ phonon combinations. Hence, we find the phonon
TABLE I. Mode assignments for the room temperature Raman spectra of SSTO.

\begin{tabular}{|c|c|c|c|}
\hline \multirow{2}{*}{$\begin{array}{l}\text { Position } \\
\omega_{0}\left(\mathrm{~cm}^{-1}\right)\end{array}$} & \multirow{2}{*}{$\begin{array}{l}\text { Zone center mode } \\
\text { assignment SSTO }\end{array}$} & \multicolumn{2}{|c|}{$\begin{array}{l}\text { Determined single-phonon } \\
\text { energies at zone boundary }\end{array}$} \\
\hline & & Phonon & Energy $\left(\mathrm{cm}^{-1}\right)$ \\
\hline \multirow[t]{3}{*}{77.51} & $\mathrm{TO}_{2}-\mathrm{TA}$ & $\mathrm{TO}_{1}$ & 111 \\
\hline & $\mathrm{TO}_{2}-\mathrm{TO}_{1}$ & & 122.45 \\
\hline & & & 118.56 \\
\hline \multirow[t]{3}{*}{174.9} & $\mathrm{TO}_{2}{ }^{\mathrm{a}}$ & $\mathrm{TA}$ & 122.45 \\
\hline & & & 111.05 \\
\hline & & & 118.25 \\
\hline \multirow[t]{3}{*}{244.9} & $2 \mathrm{TA}$ & $\mathrm{TO}_{2}$ & 191.45 \\
\hline & $2 \mathrm{TO}_{1}$ & & 188.56 \\
\hline & $\mathrm{TO}_{1}+\mathrm{TA}$ & & \\
\hline \multirow[t]{3}{*}{299.6} & $\mathrm{TO}_{2}+\mathrm{TA}$ & $\mathrm{TO}_{4}$ & 501.15 \\
\hline & $\mathrm{TO}_{2}+\mathrm{TO}_{1}$ & & 512.05 \\
\hline & $\mathrm{TO}_{4}-\mathrm{TO}_{2}$ & & \\
\hline \multirow[t]{3}{*}{382.9} & $\mathrm{TO}_{4}$-TA & $\mathrm{TO}_{3}$ & 334.8 \\
\hline & $\mathrm{TO}_{4}-\mathrm{TO}_{1}$ & & \\
\hline & $2 \mathrm{TO}_{2}$ & & \\
\hline \multirow[t]{2}{*}{619.4} & $\mathrm{TO}_{4}+\mathrm{TA}$ & & \\
\hline & $\mathrm{TO}_{4}+\mathrm{TO}_{1}$ & & \\
\hline 669.6 & $2 \mathrm{TO}_{3}$ & & \\
\hline 724.5 & $\mathrm{TO}_{4}+\mathrm{TO}_{2}$ & & \\
\hline 788.1 & $\mathrm{LO}_{4}{ }^{\mathrm{a}}$ & & \\
\hline
\end{tabular}

${ }^{a}$ First order zone center optical phonon.

energies to be $\mathrm{TA}=\mathrm{TO}_{1}=114 \mathrm{~cm}^{-1}$ (close to the estimate of $125 \mathrm{~cm}^{-1}$ above) and $\mathrm{TO}_{2}=194 \mathrm{~cm}^{-1}$. The mode obtained at $623 \mathrm{~cm}^{-1}$ is due to the superposition of $\mathrm{TO}_{4}$ and TA, or of $\mathrm{TO}_{4}$ and $\mathrm{TO}_{1}$; and that obtained at $722 \mathrm{~cm}^{-1}$ is due to superposition of $\mathrm{TO}_{4}$ and $\mathrm{TO}_{2}$. The result that we obtained from lattice dynamical studies is similar to that obtained by Nilsen and Skinner. ${ }^{12}$ The numerical values derived for energy of $\mathrm{TO}_{1}, \mathrm{TA}, \mathrm{TO}_{2}, \mathrm{TO}_{4}$, and $\mathrm{TO}_{3}$ single phonons are given in Table I.

In the room temperature Raman spectra (Fig. 3), there are two more relatively sharp modes at 174.9 and $788.1 \mathrm{~cm}^{-1}$ that are not assignable to second-order scattering. We ascribe the former to $\mathrm{TO}_{2}$ and the later to $\mathrm{LO}_{4}$ firstorder Raman scattering modes ${ }^{13,14}$ originating from lowering of the crystal symmetry with the silicon impurity doped STO ceramics as a result of breaking of inversion symmetries and it is consistent with the findings from the XRD analysis. The "silent" $\mathrm{TO}_{2}$ and $\mathrm{LO}_{2}$ modes are both at $173 \mathrm{~cm}^{-1}$ in undoped STO, as first determined by the early electric-field induced Raman scattering studies ${ }^{15,16}$ by Worlock and Fleury. The appearance of $\mathrm{TO}_{2}$ and $\mathrm{LO}_{4}$ distinct first-order modes in SSTO was confirmed by recording its vibrational spectra at $150 \mathrm{~K}$ (Fig. 3) where no structural phase transition is expected and is considered as evidence of the existence of polar nano-regions in the material. ${ }^{17}$ The smaller effective ionic radii of sixfold coordinated $\mathrm{Si}^{4+}(0.400 \AA)$ compared with that of $\mathrm{Ti}^{4+}(0.605 \AA)^{18}$ can cause $\mathrm{Si}^{4+}$ to adopt offcentered positions and create electric dipoles around which polar nano-regions can develop. It was found that the eigenfrequencies of the three TO optical phonon modes $\left(\mathrm{TO}_{1} \mathrm{TO}_{2}\right.$ and $\left.\mathrm{TO}_{4}\right)^{19}$ that characterize dielectric response of SSTO are 
lower (softened) in comparison with that of pure STO. ${ }^{12}$ It is worth to note that no Raman modes corresponding to silicon dioxide $^{20}$ that may originate due to phase segregation were observed in the experimental window indicating the phase purity of this oxide compound. Four additional phonon modes were emerged at $37,144,447$, and $545 \mathrm{~cm}^{-1}$ on cooling down the sample to $125-80 \mathrm{~K}$ range (indicated by $*$ in Fig. 3) and indicated the possible phase transition of cubic SSTO to tetragonal system.

\section{Diffuse reflectance spectroscopy}

Figure 4 shows the diffuse-reflectance spectra of about $1 \mathrm{~mm}$ thick opaque SSTO polycrystalline ceramic sample. The Kebulka-Munk function $F$ was deduced from the spectral reflectance data using the Kebulka-Munk relation $F=(1-R)^{2} / 2 R$, where $\mathrm{R}$ is the percentage of reflected light. As shown in the $\operatorname{plot}^{21}$ of $(\mathrm{dF} / \mathrm{d} \lambda)$ versus $\lambda$ given in the inset of Fig. 4, diffuse reflectance spectra exhibit an absorption threshold with an inflection point at $\sim 379 \mathrm{~nm}$ $(\sim 3.27 \mathrm{eV})$ that we attribute to the optical bandgap. It can be noted that the silicon substitution has increased the bandgap of STO negligibly from $3.25 \mathrm{eV} .{ }^{22}$ The moderately large bandgap will be of interest for electronic device applications if the material has sufficiently enhanced dielectric and electrical characteristics.

\section{Dielectric spectroscopy}

Figure 5 shows the variation in the real part of dielectric permittivity $\left(\varepsilon^{\prime}\right)$ and the loss tangent $(\tan \delta)$ of SSTO as a function of frequency $(100 \mathrm{~Hz}$ to $1 \mathrm{MHz})$ in the temperature range of $100 \mathrm{~K}$ to $500 \mathrm{~K}$ in a semi-logarithmic scale. The room-temperature dielectric constant and the dielectric loss were found to be $\sim 425$ and $\sim 0.03$, respectively, at a frequency of $10 \mathrm{kHz}$. The dielectric constant obtained was higher than the value for the pure STO sintered ceramic (about 300$)^{23}$ and is in good agreement with the observation of softened $\mathrm{TO}_{2}$ mode in SSTO room temperature Raman spectra. The

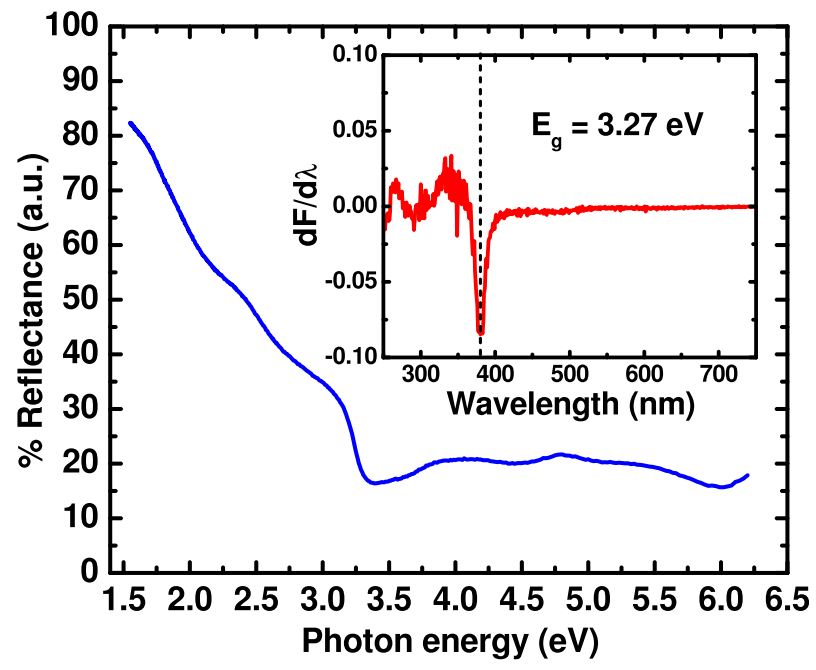

FIG. 4. Diffuse-reflectance spectra of SSTO bulk sample. The vertical line in the first derivative of Kebulka-Munk function with respect to wavelength $(\mathrm{dF} / \mathrm{d} \lambda)$ versus wavelength $(\lambda)$ plot, given in the inset, indicates the inflection point, which represents the bandgap of the material at $\sim 379 \mathrm{~nm}(\sim 3.27 \mathrm{eV})$.

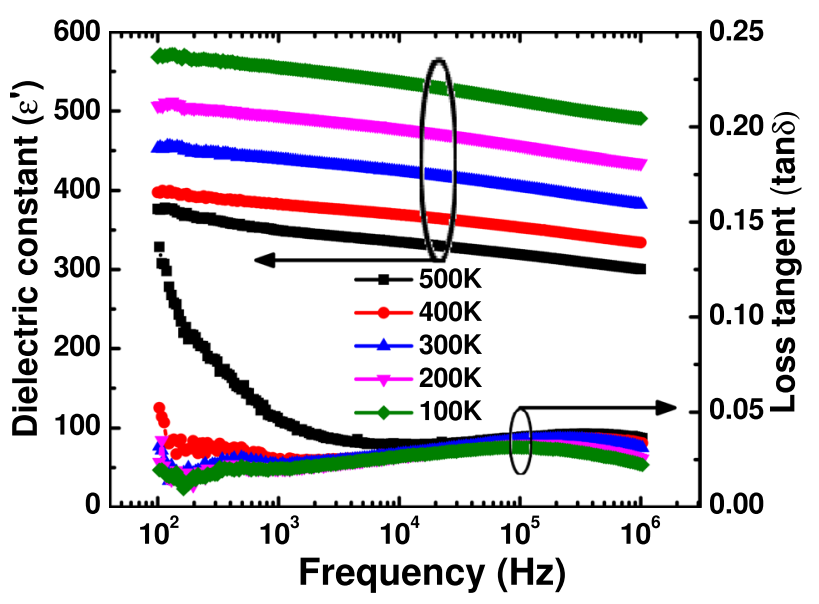

FIG. 5. Variation of dielectric constant $\left(\varepsilon^{\prime}\right)$ and dissipation factor $(\tan \delta)$ of SSTO ceramics as a function of frequency at different temperatures.

dielectric loss is very low and is nearly 0.03 below $500 \mathrm{~K}$. These features are beneficial for the DRAM application. The dielectric value decreases as the frequency increases and the trend is consistent for all temperatures. However, the tangent loss shows inconsistency for the high temperature values which is explained in the following paragraph.

Figure 6 depicts the variation of dielectric constant and loss tangent with temperature in the $100 \mathrm{~Hz}$ to $1 \mathrm{MHz}$ frequency range. The dielectric constant increases systematically with decrease in temperature for all frequencies resembling the quantum paraelectric behavior of pure STO where the dielectric constant remains large and roughly constant for temperatures $\sim 0 \mathrm{~K}^{24}$ The loss tangent remains almost constant for the ceramic in the $100 \mathrm{~K}$ to $300 \mathrm{~K}$ temperature range. However, there is an onset of high temperature dielectric relaxation process beyond $300 \mathrm{~K}$ that originates mainly from the presence of polar nano-regions in the sample as already observed from Raman studies. ${ }^{17}$ It can be noted that for a given frequency the loss tangent tends to peak ahead of the dielectric constant and the rising (peaking) of both these parameters shifts to higher temperature with increasing frequency, suggestive of features of high temperature relaxor ferroelectricity.

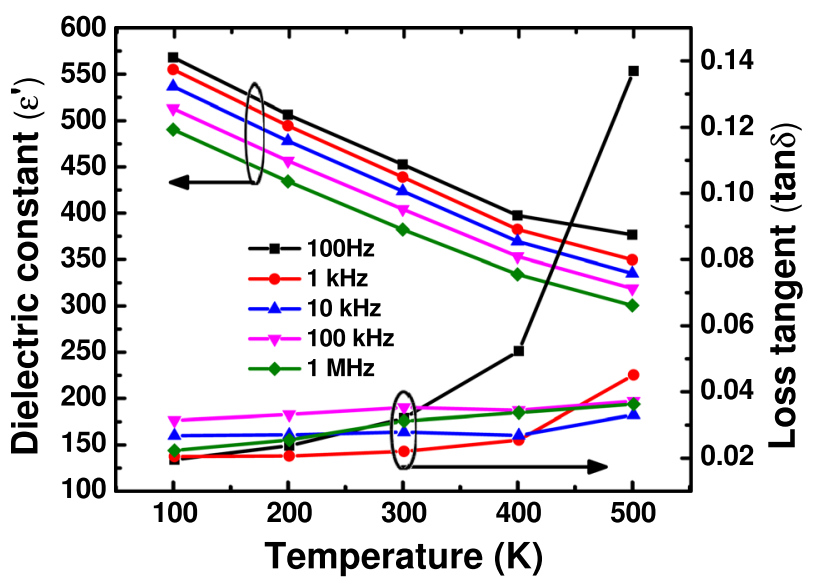

FIG. 6. Temperature dependence of dielectric constant $\left(\varepsilon^{\prime}\right)$ and loss tangent $(\tan \delta)$ of SSTO ceramics at various frequencies. 


\section{E. AC and DC conductivities}

The frequency dependence $(100 \mathrm{~Hz}-1 \mathrm{MHz})$ of the ac conductivity $\left(\sigma_{a c}\right)$ of SSTO ceramics (Fig. 7) in the 100 to $500 \mathrm{~K}$ temperature range was evaluated using the data obtained from capacitance-tangent loss measurements and by using the relation $\sigma_{a c}=\omega \varepsilon_{0}\left(\varepsilon^{\prime}\right)^{2} \tan \delta$. This measurement has significant implications on the application of this material in ac electronic devices such as MOSFET and DRAM. Figure 7 demonstrates the phenomenon of almost temperature-independent monotonic rise in dynamic conductivity with increase in frequency and suggests that the ac conductivity must be due to bound carriers trapped in the sample-not due to free carriers. Only for the highest temperature $500 \mathrm{~K}$ does $\sigma_{a c}$ exhibit a low frequency dc plateau. A power-law behavior, $\sigma_{a c} \propto A \omega^{n}$ is observed for higher frequencies. Dynamic conductivity shows the typical features of universal dynamic response (UDR) and obeys the power law $^{25}$

$$
\sigma_{a c}=\sigma_{d c}+A \omega^{n}
$$

where $\sigma_{d c}$ is the frequency independent dc conductivity, $\omega=2 \pi \mathrm{f}$ is the angular frequency, and $A$ and $n(0<n<1)$ are material-dependent constants. In our case, the numerical value of temperature-independent frequency exponent $n$ where $\sigma_{a c} \sim A \omega^{n}$ is estimated to be about 0.97 and the transport is dominated by hopping in finite clusters. In Fig. 7 (inset) below a frequency of $10^{4} \mathrm{rad} / \mathrm{s}$ a shoulder is present for higher temperatures, which can be identified as due to high temperature dielectric relaxation phenomenon as observed in the temperature dependence of $\varepsilon^{\prime}$ in Fig. 6 .

The temperature dependence of both $\sigma_{a c}$ and $\sigma_{d c}$ was measured while cooling the sample from $500 \mathrm{~K}$ to $100 \mathrm{~K}$. Figure 8 shows the dynamic conductivity (log scale) plots as a function of $1000 / \mathrm{T}\left(\mathrm{K}^{-1}\right)$ for $100 \mathrm{~Hz}, 1 \mathrm{kHz}, 10 \mathrm{kHz}$, $100 \mathrm{kHz}$, and $1 \mathrm{MHz}$ frequencies and illustrates that there is a significant amount of conductivity dispersion with frequency, implying that the effective masses of the charged particles responding to the applied field are heavy. There is

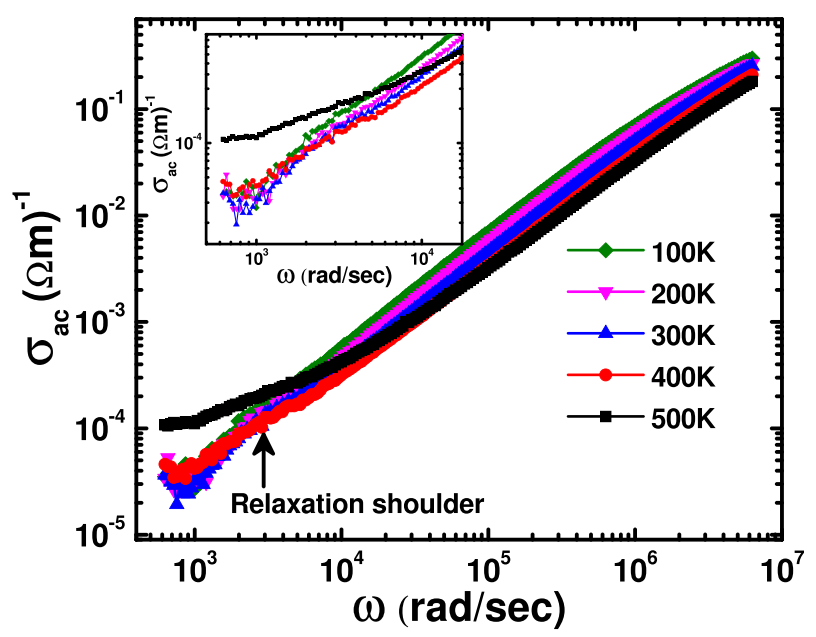

FIG. 7. AC conductivity $\left(\sigma_{a c}\right)$ of SSTO polycrystalline ceramics as a function of frequency at several temperatures from $100 \mathrm{~K}$ to $500 \mathrm{~K}$ in steps of $100 \mathrm{~K}$. The magnified relaxation shoulder is shown in the inset.

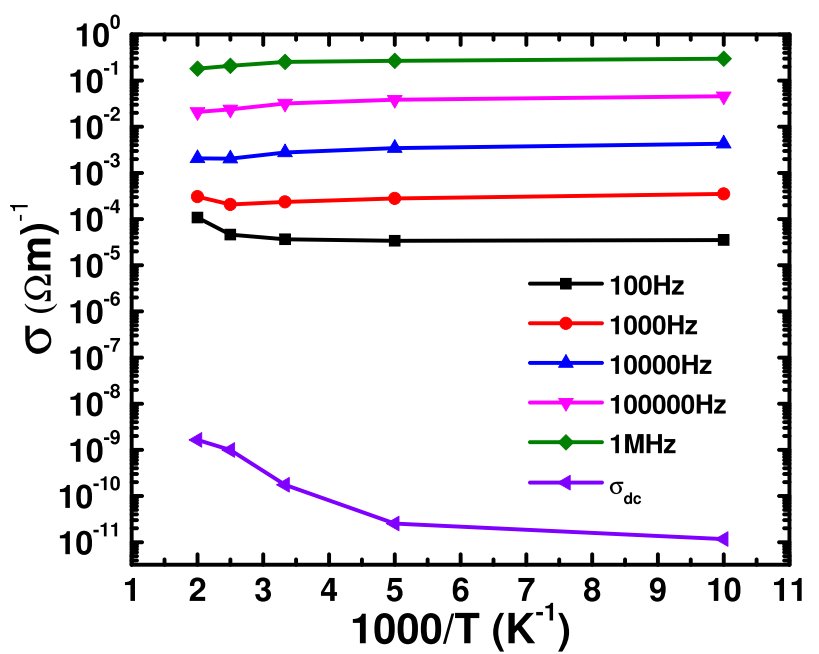

FIG. 8. Electrical conductivity $\left(\sigma_{a c}\right.$ and $\sigma_{d c}$ ) of SSTO ceramics as a function of reciprocal temperature.

no considerable variation in $\sigma_{a c}$ with temperature up to $300 \mathrm{~K}$, and above that it slowly increases only for frequencies $\leq 1 \mathrm{kHz}$. The observed increase in $\sigma_{a c}$ with frequency suggests that the thermal activation must be due to hopping of bound carriers trapped in localized states in the bandgap of the insulator. The numerical value of room temperature $\mathrm{dc}$ conductivity is $1.75 \times 10^{-10}$ (SI units), and this parameter is about 5-7 orders of magnitude lower than that of the ac conductivity in the experimental temperature range. This is due to the fact that $\sigma_{d c}$ is determined by the most difficult transition in complete percolation paths between the electrodes, while the $\sigma_{a c}$ is determined by the easiest local movement of the charges. Thermally activated $\sigma_{d c}$ increases with temperature, however, even extrapolation of ac values to zero frequency does not yield the measured dc response. It is anticipated in such systems that large contributions arise at very low frequencies $(\mathrm{Hz}$ to $\mathrm{kHz}$ ), from mobile ions including oxygen vacancies, domain walls, or other topological defects, and these cannot be anticipated quantitatively from higher frequency ac measurements. The rise in $\sigma_{d c}$ may originate from thermally generated carriers in the sample, resulting in semiconductor-type conduction. The linearity between $\log \sigma_{d c}$ and $1000 / \mathrm{T}$ suggests the validity of the Arrhenius equation

$$
\sigma_{d c}=\sigma_{0} \exp -\left(E_{\text {cond }} / k_{B} T\right),
$$

where $\sigma_{0}$ is a pre-exponential term, $E_{\text {cond }}$ is the activation energy for conduction in $\mathrm{eV}, k_{B}$ is the Boltzmann constant $\left(8.617 \times 10^{-5} \mathrm{eV} / \mathrm{K}\right)$, and $T$ is the absolute temperature. The average activation energy calculated in the $200-500 \mathrm{~K}$ temperature range using Eq. (2) is $123 \mathrm{meV}$; this low numerical value suggests that electronic charge carriers are predominant in the electrical conduction. The pre-exponential factor $\sigma_{0}$ was estimated to be $2.83 \times 10^{-8}(\Omega \mathrm{m})^{-1}$. There is a change in slope (decrease in $E_{\text {cond }}$ ) for lower temperatures $(\leq 200 \mathrm{~K})$, which is indicative of a transition from a hopping transport to conduction in semi-metallic extended states and could be associated with the expected cubic to tetragonal phase transition in this temperature range. ${ }^{26}$ 


\section{F. Linear dielectric response}

Figure 9 shows the electric field dependency of dielectric constant of SSTO sample configured in Pt/SSTO/Pt MIM capacitor structure at various temperatures $(100-500 \mathrm{~K})$. A field- independent dielectric response and little hysteresis were observed for this electronic material while sweeping the voltage (electric field) from $-40 \mathrm{~V}(-38.8 \mathrm{kV} / \mathrm{m})$ to $+40 \mathrm{~V}$ $(+38.8 \mathrm{kV} / \mathrm{m})$. The temperature dependence $(100-500 \mathrm{~K})$ of dielectric constant measured at $100 \mathrm{kHz}$ showed considerable change with respect to the room temperature value $(-21 \%$ and $+27 \%$ ), as depicted in the inset (a) of Fig. 9. The temperature coefficient of capacitance $\alpha_{T}$, another figure of merit of the dielectric capacitor devices, is given by the relation

$$
\frac{C_{T}-C_{0}}{C_{0}}=T \alpha_{T},
$$

where $C_{T}$ and $C_{0}$ are the capacitance values at a particular temperature $(T)$ and at room temperature $(300 \mathrm{~K})$, respectively. The numerical values of $\alpha_{T}$ for the SSTO sample were determined to be $-424,-302,678$, and $2699 \mathrm{ppm} / \mathrm{K}$ at temperatures 500, 400, 200, and $100 \mathrm{~K}$, respectively, at $100 \mathrm{kHz}$ ac signal frequencies. The capacitance versus temperature plot [inset (b) of Fig. 9] provided a negative slope of $9.48 \times 10^{-2} \mathrm{pF} / \mathrm{K}$. The inset (c) of Fig. 9 shows the charge storage density $\left(\mathrm{Q}_{\mathrm{c}}=\varepsilon_{0} \varepsilon^{\prime} E\right)$ of the sample as a function of applied electric field and demonstrates the linear dependence between these two parameters (paraelectric behavior) with a slope, absolute permittivity, of $0.3561 \times 10^{-8} \mathrm{~F} / \mathrm{m}$. An encouraging charge storage density value of $\sim 0.14 \mathrm{fC} / \mu \mathrm{m}^{2}$ was estimated for an applied electric field of $\sim 39 \mathrm{kV} / \mathrm{m}$. The measured quality factor, the ratio of the average energy stored to the energy dissipated per cycle, $Q=(1 / \tan \delta)$ was well above the limiting value of 20 for DRAM applications at all the measured frequencies and temperatures (data not

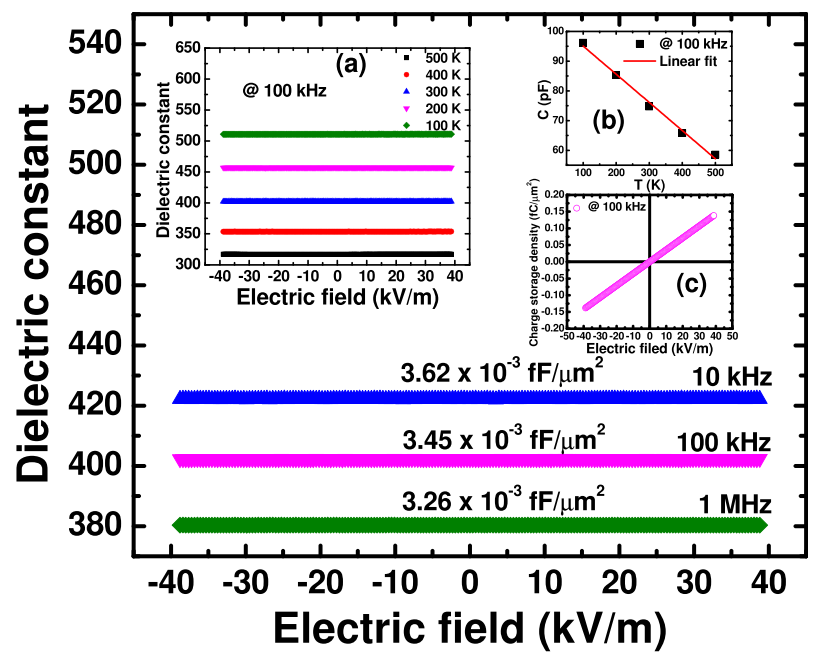

FIG. 9. Electric field dependency of relative permittivity of Pt/SSTO/Pt MIM capacitors at $10 \mathrm{kHz}, 100 \mathrm{kHz}$, and $1 \mathrm{MHz}$ evaluated from data obtained from C-V measurements. The inset (a) shows the linear dielectric response of the SSTO sample in a wide range of temperature $(100-500 \mathrm{~K})$. The inset (b) depicts the inverse variation of capacitance with temperature. Charge storage density versus the applied electric field characteristic for the sample recorded at $100 \mathrm{kHz}$ signal frequency is given in the inset (c). shown). The linear/flat dielectric response of SSTO reveals the applicability of this material for MIM stacked capacitors, such as radio frequency (RF) coupling and bypass capacitors in oscillators and resonator circuits, filter and analog capacitors in analog/mixed-signal (AMS) circuits, decoupling capacitors for microprocessors (MPUs), and storage capacitors in dynamic random access memory (DRAM) and embedded DRAM (eDRAM)/logic devices.

\section{G. Leakage current behavior}

Electronic materials need to posses not only a high charge storage density but also a low leakage current density for their successful application in high performing and reliable logic (MOSFET) and memory (DRAM, RRAM, etc.) devices. The current density $(\mathrm{J})$ versus electric field $(\mathrm{E})$ characteristics of SSTO sample measured in the temperature range of $100 \mathrm{~K}$ to $500 \mathrm{~K}$ for positive bias are shown in Fig. 10. The leakage current density of the symmetric MIM (Pt/SSTO/Pt) stack exhibited moderate temperature ( 0.66 to $118 \mathrm{nA} / \mathrm{cm}^{2}$ at the maximum electric field of $\left.8.7 \mathrm{kV} / \mathrm{cm}\right)$ and field dependence $\left(3.22\right.$ to $13.04 \mathrm{nA} / \mathrm{cm}^{2}$ at $\left.300 \mathrm{~K}\right)$ and the observed exceptionally very low numerical value of $13 \mathrm{nA} / \mathrm{cm}^{2}$ at $8.7 \mathrm{kV} / \mathrm{cm}$ at room temperature confirms the good insulator behavior of the material. The data at comparatively low electric field were found not to fit thermionic emission (Schottky or Poole-Frenkel), trap-assisted tunneling, or space charge limited conduction mechanisms. Based on these analyses, we conclude that the charge transport is dominated by a bulk conduction mechanism in which at low electric fields the Pt/SSTO interface, which possesses a larger Schottky barrier height blocks injection of electrons from the higher work function Pt bottom electrode in to the bulk dielectric material.

\section{CONCLUSIONS}

Polycrystalline samples of silicon substituted STO were successfully synthesized by a solid-state reaction technique and well characterized using XRD, Raman spectroscopy, EDX, SEM, and XRF techniques. The distortion in the cubic

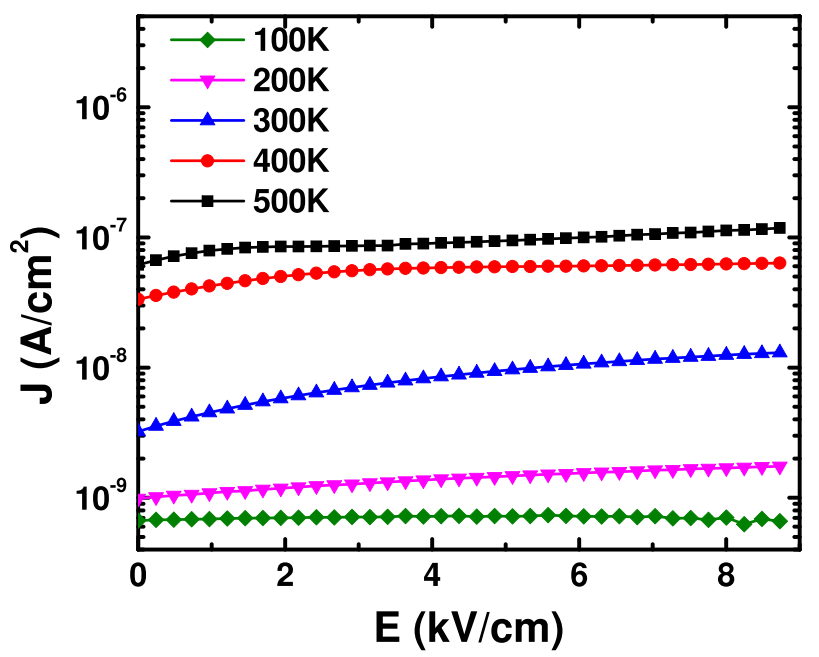

FIG. 10. Leakage current density of Pt/SSTO/Pt symmetric MIM capacitor at various temperatures. 
lattice, as a result of breaking of inversion symmetry, and/or translation symmetries due to doping was revealed by the asymmetry and splitting in the XRD spectra and the observation of the first order $\mathrm{TO}_{2}$ and $\mathrm{LO}_{4}$ modes in Raman spectra. In combination with the high linear dielectric constant of $\sim 400$ and very low dielectric loss of $\sim 0.03$ at $100 \mathrm{kHz}$ and the large observed bandgap $\mathrm{E}_{\mathrm{g}}$ of $3.27 \mathrm{eV}$ near ambient conditions, our studies reveal the fundamental physics and materials science of the SSTO electroceramics and its potential application as a high-k dielectric for the materialsenabled scaling of the next generation of silicon technology devices.

\section{ACKNOWLEDGMENTS}

Financial support from NSF Grant No. NSF-RII0701525 was acknowledged. S.D. is thankful to DOD for doctoral fellowship under Grant No. W911NF-11-1-0204. S. P.P. is grateful to NSF for financial assistance under Grant No: NSF-EFRI RESTOR \# 1038272.

${ }^{1}$ R. C. Neville, B. Hoeneisen, and C. A. Mead, J. Appl. Phys. 43, 2124 (1972).

${ }^{2}$ A. K. Tagantsev, V. O. Sherman, K. F. Astafiev, J. Venkatesh, and N. Setter, J. Electroceram. 11, 5 (2003).

${ }^{3}$ M. Cardona, Phys. Rev. 140(2A), A651 (1965).

${ }^{4}$ C. S. Hwang, S. O. Park, C. S. Kang, H. Cho, H. Kang, S. T. Ahn, and M. Y. Lee, Jpn. J. Appl. Phys., Part 1 34, 5178 (1995).

${ }^{5}$ S. Stille, C. Baeumer, S. Krannich, C. Lenser, R. Dittmann, J. Perlich, S. V. Roth, R. Waser, and U. Klemradt, J. Appl. Phys. 113, 064509 (2013).

${ }^{6}$ R. A. McKee, F. J. Walker, and M. F. Chisholm, Phys. Rev. Lett. 81, 3014 (1998).
${ }^{7}$ V. V. Lemanov, E. P. Smirnova, P. P. Syrnikov, and E. A. Tarakanov, Phys Rev B 54(5), 3151 (1996).

${ }^{8}$ J. G. Bednorz and K. A. Müller, Phys. Rev. Lett. 52(25), 2289 (1984).

${ }^{9}$ W. Luo, W. Duan, S. G. Louie, and M. L. Cohen, Phys. Rev. B 70(21), 214109 (2004).

${ }^{10}$ F. A. Rabuffetti, H.-S. Kim, J. A. Enterkin, Y. Wang, C. H. Lanier, L. D. Marks, K. R. Poeppelmeier, and P. C. Stair, Chem. Mater. 20, 5628 (2008).

${ }^{11}$ C. H. Perry, J. H. Fertel, and T. F. McNelly, J. Chem. Phys. 47, 1619 (1967).

${ }^{12}$ W. G. Nilsen and J. G. Skinner, J. Chem. Phys. 48, 2240 (1968).

${ }^{13}$ A. A. Sirenko, I. A. Akimov, J. R. Fox, A. M. Clark, H.-C. Li, W. Si, and X. X. Xi, Phys. Rev. Lett. 82(22), 4500 (1999).

${ }^{14}$ H. Vogt and G. Rossbroich, Phys. Rev. B 24, 3086 (1981).

${ }^{15}$ P. A. Fleury and J. M. Worlock, Phys. Rev. 174, 613 (1968).

${ }^{16}$ J. F. Scott, P. A. Fleury, and J. M. Worlock, Phys. Rev. 177, 1288 (1969).

${ }^{17}$ R. Ranjan, R. Hackl, A. Chandra, E. Schmidbauer, D. Trots, and H. Boysen, Phys. Rev. B 76, 224109 (2007).

${ }^{18}$ R. D. Shannon, Acta Crystallogr., Sect. A: Cryst. Phys., Diffr., Theor. Gen. Crystallogr. 32, 751 (1976).

${ }^{19}$ J. Han, F. Wan, Z. Zhu, and W. Zhang, Appl Phys Lett 90(3), 031104 (2007).

${ }^{20}$ P. Gillet, A. Le Cleach, and M. Madon, J. Geophys. Res. 95, 21635, doi:10.1029/JB095iB13p21635 (1990).

${ }^{21}$ S. P. Pavunny, Y. Sharma, S. Kooriyattil, S. Dugu, R. K. Katiyar, J. F. Scott, and R. S. Katiyar, Appl. Phys. Lett. 106(11), 112902 (2015).

${ }^{22}$ K. van Benthem, C. Elsässer, and R. H. French, J. Appl. Phys. 90(12), 6156 (2001)

${ }^{23}$ Landoldt-Börnstein: Numerical Data and Functional Relationships in Science and Technology, edited by O. Madelung (Springer, Berlin, 1982), Chap. 17a.

${ }^{24}$ K. A. Miiller, W. Berlinger, and E. Tosatti, Z. Phys. B 84, 277 (1991).

${ }^{25}$ A. K. Jonscher, Dielectric Relaxation in Solids (Chelsea Dielectrics Press, London, 1983).

${ }^{26}$ N. F. Mott, E. A. Davis, and R. A. Street, Philos. Mag. 32, 961 (1975). 